\title{
MiR-423-5p downregulates osteoblastic differentiation and cell viability by targeting SMAD3 in non-traumatic osteonecrosis
}

\author{
Ju Zheng ${ }^{1}$, Xuegang Yan ${ }^{1}$, Yun Zeng ${ }^{2 *}$ \\ ${ }^{1}$ Department of Orthopaedics, Medicalservice Community of People's Hospital of Fenghuaningbo, Ningbo City, Zhejiang \\ Province 315500, ${ }^{2}$ Department of Orthopaedics, Dongfeng Hospital Affiliated with Hubei University of Medicine, Shiyan, Hubei \\ Province 442008, China
}

*For correspondence: Email: yzeng666@163.com; Tel: +86-719-8272457

Sent for review: 3 December 2020

Revised accepted: 26 February 2021

\begin{abstract}
Purpose: To investigate the potential role of miR-423-5p in osteoblastic differentiation of non-traumatic osteonecrosis of the femoral head (ONFH).

Methods: MiR-423-5p levels in bone marrow samples from ONFH and osteoarthritis (OA) patients, respectively, were evaluated using quantitative (real-time) polymerase chain reaction (qPCR). Osteoblastic differentiation was monitored using Alizarin red $S$ staining, while cell viability was determined by MTT assay in hMSC-BM. MiR-423-5p expression was also measured during osteoblastic differentiation. The underlying mechanisms were explored using TargetScan database, and a series of in vitro experiments was performed to confirm this.

Results: MiR-423-5p levels were significantly upregulated in ONFH samples $(p<0.01)$ and miR-423-5p expression significantly increased in human mesenchymal stem cells-bone marrow (hMSC-BM) after bone morphogenetic protein 2 (BMP-2) treatment. Furthermore, miR-423-5p downregulated osteoblastic differentiation and suppressed cell viability. Furthermore, SMAD3 was observed to be a downstream target of miR-423-5p via bioinformatics analysis; further in vitro experiments confirmed this.

Conclusion: MiR-423-5p downregulates osteoblastic differentiation and cell viability by targeting SMAD3 in non-traumatic osteonecrosis. Thus, MiR-423-5p may serve as a potential target for promoting osteoblastic differentiation in ONFH patients.
\end{abstract}

Keywords: MiR-423-5p, Non-traumatic osteonecrosis, Femoral head, Osteoblastic differentiation, Cell viability, SMAD3

\begin{abstract}
This is an Open Access article that uses a fund-ing model which does not charge readers or their institutions for access and distributed under the terms of the Creative Commons Attribution License (http://creativecommons.org/licenses/by/4.0) and the Budapest Open Access Initiative (http://www.budapestopenaccessinitiative.org/read), which permit unrestricted use, distribution, and reproduction in any medium, provided the original work is properly credited.

Tropical Journal of Pharmaceutical Research is indexed by Science Citation Index (SciSearch), Scopus, International Pharmaceutical Abstract, Chemical Abstracts, Embase, Index Copernicus, EBSCO, African Index Medicus, JournalSeek, Journal Citation Reports/Science Edition, Directory of Open Access Journals (DOAJ), African Journal Online, Bioline International, Open-J-Gate and Pharmacy Abstracts
\end{abstract}

\section{INTRODUCTION}

Osteonecrosis (ON) is a disease characterized by destruction of the blood supply of the femoral head [1]. ON is most common between the ages of 30 and 50 years [2]. Nontraumatic osteonecrosis of the femoral head (ONFH) is caused by increased intra-osseous pressure and abnormal formation of bone marrow fat, which subsequently lead to ischemia and osteocyte and bone-marrow cell death $[3,4]$. Treatment strategies, including core decompression and 
joint replacement surgery, are usually applied to ONFH patients [5]. However, these traditional treatments only achieve a moderate reduction in intramedullary pressure, with little clinical benefit and high cost [2].

Previous studies showed that ONFH is associated with changes in bone marrow mesenchymal stem cell (MSC)-derived osteogenic differentiation [6]. This finding suggests the possibility that reduced capacity for MSC osteoblastic differentiation or MSC viability plays an important role in ONFH.

MicroRNAs (miRNAs) are a cohort of 18-22nucleotide small noncoding RNAs that regulate thousands of target genes [7]. MiRNAs bind mainly to the 3'-untranslated region (UTR) of the target mRNA, leading to inhibition of translation or degradation of the mRNA [8]. Moreover, miRNAs are reported to play regulatory roles in many diseases, and osteoblastic-differentiationrelated miRNAs have been widely studied. For example, in nontraumatic osteonecrosis, miR-17$5 p$ affects osteoblast differentiation through downregulation of SMAD7 [9]. $\mathrm{Xu}$ WH et al reported that $\mathrm{miR}-186-5 p$ regulates osteoblast differentiation via decreased CXCL13 [10]. In addition, miR-217 was also implicated in the promotion of BMSC proliferation [11].

Previous evidence indicated that miR-423-5p levels in the serum of ONFH patients were significantly increased, which was positively correlated with femoral head collapse and negatively correlated with adiponectin level, demonstrating that miR-423-5p is probably implicated in the pathology of ONFH progression [12]. In addition, miR-423 was implicated in tumorigenesis. However, the role of miR-423-5p in the viability and osteogenic differentiation of MSCs in ONFH has not been reported.

In this study, we aimed to investigate the potential role of $\mathrm{miR}-423-5 p$ in osteoblastic differentiation in ONFH and explore the corresponding mechanisms.

\section{EXPERIMENTAL}

\section{Clinical samples}

Following the approval of the Ethics Committee of Dongfeng Hospital Affiliated with Hubei University of Medicine (Approval No. LW-2020015)[13], a cohort of bone marrow samples from nontraumatic ONFH patients and osteoarthritis (OA) patients was collected. All patients provided written informed consent.

\section{Cell culture and transfection}

HEK-293T cells and human mesenchymal stem cells-bone marrow (hMSC-BM) were used in the present study and purchased from the Type Culture Collection of the Chinese Academy of Sciences. HMSC-BM were transfected with miR423-5p mimics or miR-423-5p inhibitor using FuGENE (Roche Molecular Biochemicals, Indianapolis, IN, USA). Human SMAD3 coding sequences were cloned into the vector pcDNA3.1 (Addgene, Cambridge, MA, USA) for the construction of the SMAD3 plasmid, and transient transfection was performed with Lipofectamine $^{\mathrm{TM}} 2000$ Transfection Reagent (Invitrogen, Carlsbad, CA, USA) according to the manufacturer's instructions.

\section{Quantitative (real-time) polymerase chain reaction (qPCR)}

RNA extraction was performed using mirVana miRNA Isolation Kit (Thermo Fisher Scientific, Waltham, MA, USA) and then RNA was reverse transcribed into cDNA using TaqMan ${ }^{\mathrm{TM}}$ MicroRNA Reverse Transcription Kit (Thermo Fisher Scientific). CFX96 Real-Time PCR system (Bio-Rad, Hercules, CA, USA) was used for qPCR, and the relative expression of miR-423-5p was determined using the $2^{-\Delta \Delta C T}$ method. Primers for miR-423-5p and U6 snRNA, which served as internal control for miRNA, were designed and supplied by Guangzhou RiboBio Co., Ltd (Guangzhou, China).

\section{Western blotting}

Total proteins were harvested using RIPA lysis buffer and the concentrations were measured. Total protein $(50 \mu \mathrm{g})$ was loaded and separated in $8 \%$ SDS-polyacrylamide gels. Proteins were transferred to PVDF membranes, and the membranes were blocked with 5\% BSA. Protein immunoblots were performed with primary antibodies against SMAD3 (ab40854, 1:1000; Abcam, Cambridge, UK) and $\beta$-actin (ab8226, 1:1000; Abcam).

\section{MTT assays}

Cell viability was determined by MTT assay. HMSC-BM were cultured in Dulbecco's Modified Eagle Medium (DMEM) with $10 \%$ fetal bovine serum (Gibco, Grand Island, NY, USA) and $1 \times$ $10^{3}$ cells per well were seeded onto 96-well plates followed by addition of $10 \mu \mathrm{L} 3-(4,5-$ dimethlthiazol-2-yl)-2,5-diphenyl-

tetrazoliumbromide (MTT, $5 \mathrm{mg} / \mathrm{mL}$ ). After $4 \mathrm{~h}$, formazan was dissolved in $100 \mu \mathrm{L}$ dimethyl sulfoxide and the absorbance was measured at 
$490 \mathrm{~nm}$. All experimental sets were performed in triplicate.

\section{Osteoblastic differentiation and Alizarin red staining}

HMSC-BM were treated with BMP-2 $(100 \mathrm{ng} / \mathrm{mL})$ to induce osteoblastic differentiation. Alizarin red $S$ staining solution (Sigma-Aldrich, St. Louis, MO, USA) was used to detect the mineralized nodules. The cells were fixed with $4 \%$ paraformaldehyde and Alizarin red $S$ staining solution was added followed by incubation of cells for another $30 \mathrm{~min}$. Subsequently, the cells were washed with double-distilled $\mathrm{H}_{2} \mathrm{O}$ to remove unbound dye and then photographed.

\section{Dual-luciferase reporter assay}

Wild-type or mutant 3'-UTR of SMAD3 was cloned into firefly-luciferase-tagged pGL3 promoter vector (Promega, Madison, WI, USA) and then HEK-293T cells were transfected with 1 $\mu \mathrm{g}$ plasmid. HEK-293T cells were co-transfected with $1 \mu \mathrm{g}$ plasmid and the Renilla luciferase vector (Promega) as control. Luciferase activity was measured using a dual luciferase assay system (Promega). Results are presented as the relative value of firefly/Renilla luciferase activity.

\section{Statistical analysis}

All results are presented as mean \pm standard deviation (SD). Results were analyzed using an independent Student's $t$-test for comparison of two groups. Data analysis was performed using software GraphPad Prism 6.0 (GraphPad, San Diego, CA, USA). A value of $p<0.05$ was considered statistically significant.

\section{RESULTS}

\section{Expression of miR-423-5p significantly increased in ONFH samples or during osteoblastic differentiation}

To determine the effect of miR-423-5p on ONFH, a cohort of bone marrow samples from ONFH patients and osteoarthritis (OA) patients was collected. MiR-423-5p levels were measured using $q P C R$, and miR-423-5p was significantly upregulated in the ONFH group compared with the OA group $(p<0.01$; Figure $1 \mathrm{~A})$. To further clarify whether miR-423-5p participated in osteoblastic differentiation, hMSC-BM were treated with BMP-2 to induce osteoblastic differentiation. The results indicated that miR423-5p levels were significantly upregulated in hMSC-BM on days $4(p=0.04)$ and $6(p<0.01)$ after BMP-2 treatment (Figure $1 \mathrm{~B}$ ).
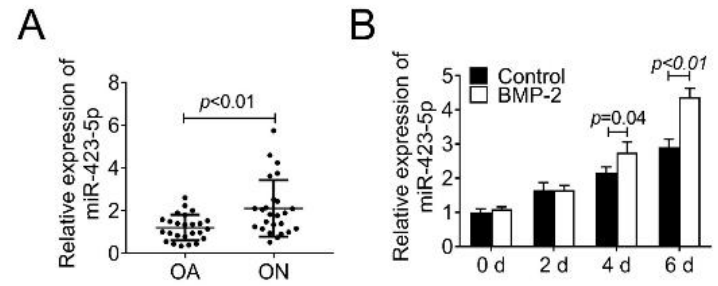

Figure 1: MiR-423-5p levels significantly increased in nontraumatic osteonecrosis of femoral head $(\mathrm{ON})$ or during osteoblastic differentiation. (A) MiR-423-5p levels were measured by $\mathrm{QPCR}$ in nontraumatic osteonecrosis of femoral head (ONFH) and osteoarthritis (OA) patients. $n=25$. (B) hMSC-BM were treated with BMP-2 $(100 \mathrm{ng} / \mathrm{mL})$ to induce osteoblastic differentiation, and miR-423-5p levels were measured using quantitative $P C R$ ( $q P C R)$ on days $0,2,4$, and 6

\section{MiR-423-5p downregulates osteoblastic differentiation and viability of hMSC-BM}

The function of miR-423-5p was further explored in hMSC-BM transfected with miR-423-5p mimics or miR-423-5p inhibitor, and relative expression of miR-423-5p was measured (Figure $2 \mathrm{~A} ; p<$ 0.01 ). First, results of MTT assay demonstrated that viability of $\mathrm{hMSC}-\mathrm{BM}$ was significantly decreased with overexpression of miR-423-5p, and cell viability was significantly increased as a result of miR-423-5p knockdown $(p<0.01$; Figure $2 \mathrm{~B}$ ). Correspondingly, fewer mineralized nodules were observed in hMSC-BM overexpressing miR-423-5p, and more mineralized nodules were observed by Alizarin red $S$ staining with miR-423-5p knockdown (Figure $2 \mathrm{C}$ ). These results suggest that miR423-5p downregulates osteoblastic differentiation and viability of hMSC-BM.
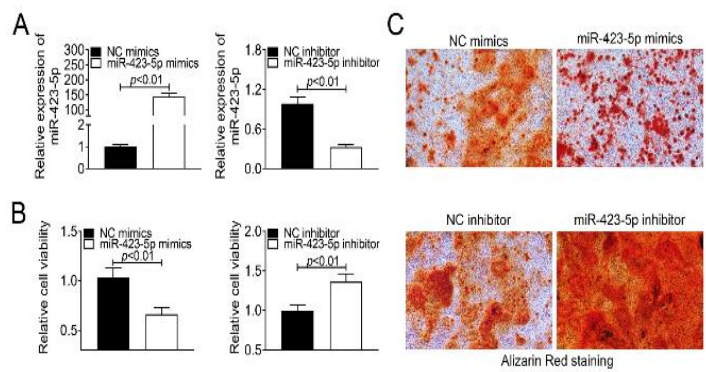

Figure 2: MiR-423-5p downregulates osteoblastic differentiation and viability of hMSC-BM. (A) HMSCBM were transfected with miR-423-5p mimics or miR423-5p inhibitor. (B) MTT assays were performed to examine viability of hMSC-BM treated with miR-423$5 p$ mimics or miR-423-5p inhibitor. (C) Mineralized nodules in hMSC-BM were stained with Alizarin red. $\mathrm{NC}$, negative control 


\section{SMAD3 is a target of miR-423-5p}

The underlying targets of miR-423-5p were predicted using the TargetScan (http://targetscan.org/) database, and the complementary sequence of miR-423-5p was identified in the 3'-UTR of SMAD3 mRNA (Figure $3 \mathrm{~A})$. The binding site was then mutated, and dual luciferase reporter assay was performed. The data suggested that luciferase activity of SMAD3 with the wild-type (WT) 3'-UTR was significantly increased as a result of miR-423-5p downregulation by treatment with miR-423-5p inhibitor whereas SMAD3 with the mutant 3'-UTR was not affected $(p<0.01$; Figure 3 B). Moreover, SMAD3 levels were significantly decreased when cells were treated with miR423-5p mimics and significantly increased in cells treated with miR-423-5p inhibitor $(p<0.01$; Figure $3 \mathrm{C}$ ). Therefore, SMAD3 is a target of miR-423-5p.
A

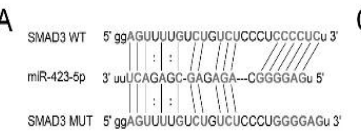

B

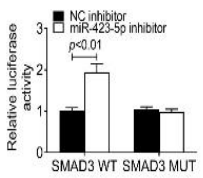

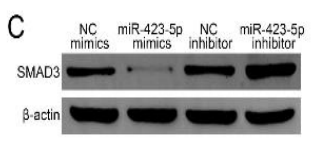

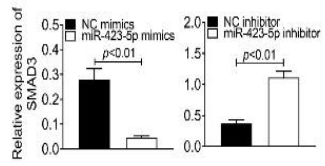

Figure 3: SMAD3 is a target of miR-423-5p. (A) The complementary sequence of miR-451a in 3'-UTR of SMAD3 mRNA and the mutated binding site. (B) Dualluciferase reporter assay was performed and luciferase activity of SMAD3 with wild-type (WT) 3'UTR or mutant (MUT) 3'-UTR was measured (C) Western analysis of SMAD3 in hMSC-B treated with miR-423-5p mimics or miR-423-5p inhibitor

\section{MiR-423-5p downregulates osteoblastic differentiation and cell viability by targeting SMAD3}

To determine whether the role of miR-423-5p in osteoblastic differentiation and cell viability is mediated by SMAD3, SMAD3 expression was significantly decreased in hMSC-BM transfected with miR-423-5p mimics to achieve miR-423-5p overexpression, and co-transfection with SMAD3 plasmid reversed this inhibitory effect of miR423-5p on SMAD3 expression $(p<0.01$; Figure 4 A). Viability of hMSC-BM was significantly decreased and SMAD3 expression was significantly downregulated as a result of miR423-5p overexpression, but this inhibitory effect on cell viability and SMAD3 expression was abolished by transfecting with SMAD3 plasmid to rescue SMAD3 expression $(p<0.01$; Figure 4 $B)$. Mineralized nodules were stained using
Alizarin red $\mathrm{S}$ and fewer mineralized nodules were observed owing to miR-423-5p overexpression, but this inhibitory effect on mineralized nodule formation was rescued by cotransfecting with SMAD3 plasmid (Figure $4 \mathrm{C}$ ). Collectively, miR-423-5p downregulates osteoblastic differentiation and cell viability by targeting SMAD3.

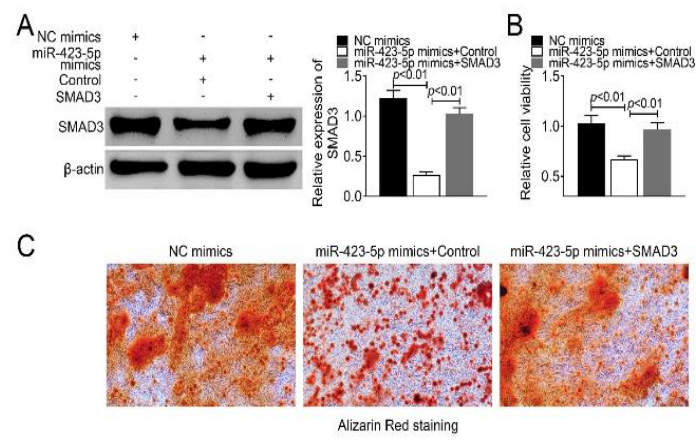

Figure 4: MiR-423-5p downregulates osteoblastic differentiation and cell viability by targeting SMAD3. (A) Western analysis of SMAD3 expression in hMSCBM treated with miR-423-5p mimics alone or in combination with SMAD3 plasmid. (B) Viability of hMSC-BM was determined using MTT assays. (C) Mineralized nodules were stained with Alizarin red in hMSC-BM treated with miR-423-5p mimics alone or in combination with SMAD3 plasmid

\section{DISCUSSION}

Femoral head osteonecrosis may be divided into two types: traumatic osteonecrosis of the femoral head and ONFH [2]. There are many causes of nontraumatic femoral head osteonecrosis, such as long-term hormone use, excessive alcohol consumption, obesity, diseases of the blood, osteoporosis, bone metabolic disorders, abnormal formation of bone marrow fat, and vascular disease [14]. Previous studies have disclosed that abnormal MSC differentiation leads to reduced osteogenic differentiation in ONFH patients [6]. In addition, accumulated evidence has demonstrated that many miRNAs, including miR-17-5p [9], miR-186-5p [10], miR217 [11], and miR-596 [15] are involved in MSC differentiation in ONFH patients. In the current study, miR-423-5p levels were significantly increased in ONFH samples or during osteoblastic differentiation. Moreover, miR-423$5 p$ downregulates osteoblastic differentiation and viability of hMSC-BM and regulates osteoblastic differentiation by directly targeting SMAD3.

Previous studies of miR-423-5p mainly focused on its effect on tumorigenesis. miR-423-5p was identified as a tumor suppressor in the tumorigenesis of ovarian cancer [16], but acts as 
an oncogene in lung adenocarcinoma and promotes metastasis to the brain [17]. In addition, hepatic NFE2/miR-423-5p signaling inhibits the FAM3A-ATP-Akt signaling pathway and then mediates the progress of type 2 diabetes and non-alcoholic fatty liver diseases (NAFLD) [18]. Moreover, miR-423-5p levels in the serum of ONFH patients is significantly increased, which is positively correlated with femoral head collapse and negatively correlated with adiponectin level, suggesting that miR-423$5 p$ may be involved in regulation of ONFH progression [12]. Therefore, the effect of miR423-5p on the viability and differentiation of MSCs in ONFH was evaluated in the current study. Surprisingly, miR-423-5p downregulates osteoblastic differentiation and viability of hMSCBM. As a member of the SMAD family, SMAD3 has a variety of biological functions. Previous studies have shown that SMAD3 is involved in osteoblastic differentiation. For example, the expression of Runx2 was significantly decreased due to SMAD3 knockdown, and loss of SMAD3 altered the cell fate of MSCs [19]. Long noncoding RNA H19 has been reported to have a role in the promotion of osteoblastic differentiation via the miR-675/TGF$\beta 1 / S M A D 3 / H D A C$ axis [20]. In addition, miR-596 and miR-708 aggravate steroid-induced ONFH by targeting SMAD3 [15,21]. In the current study, SMAD3 was shown to be a target of miR-423-5p and involved in the regulation of osteoblastic differentiation and viability of hMSC-BM.

In the present study, fewer mineralized nodules were observed in the miR-423-5p mimics group, and this inhibitory effect of miR-423-5p on mineralization was rescued by co-transfecting cells with SMAD3 plasmid to achieve SMAD3 overexpression. However, the role of SMAD3 in osteoblastic differentiation in ONFH patients has not been elucidated and the downstream target genes of SMAD3 were not identified in this study.

\section{CONCLUSION}

The findings of this study indicate that miR-423$5 p$ exerts an ameliorative effect on osteoblastic differentiation in ONFH patients. Thus, miR-423$5 p$ may serve as a potential target for promoting osteoblastic differentiation in ONFH patients.

\section{DECLARATIONS}

\section{Acknowledgement}

This work was supported by the Shiyan City Science and Technology Research and Development Programme (Grant no. HS149147).

\section{Competing interests}

There is no conflict of interest to disclose.

\section{Contribution of authors}

We declare that this work was done by the authors named in this article and all liabilities pertaining to claims relating to the content of this article will be borne by the authors. Ju Zheng designed the study, supervised the data collection; Xuegang Yan analyzed the data, interpreted the data; Yun Zeng prepared the manuscript for publication and reviewed the draft of the manuscript. All authors have read and approved the manuscript.

\section{Open Access}

This is an Open Access article that uses a funding model which does not charge readers or their institutions for access and distributed under the terms of the Creative Commons Attribution License (http://creativecommons.org/licenses/by/ 4.0) and the Budapest Open Access Initiative (http://www.budapestopenaccessinitiative.org/rea d), which permit unrestricted use, distribution, and reproduction in any medium, provided the original work is properly credited.

\section{REFERENCES}

1. Wang C, Peng J, Lu S. Summary of the various treatments for osteonecrosis of the femoral head by mechanism: A review. Exp Ther Med 2014; 8(3): 700706.

2. Petek D, Hannouche D, Suva D. Osteonecrosis of the femoral head: pathophysiology and current concepts of treatment. EFORT Open Rev 2019; 4(3): 85-97.

3. Liu F, Wang W, Yang L, Wang B, Wang J, Chai W, Zhao D. An epidemiological study of etiology and clinical characteristics in patients with nontraumatic osteonecrosis of the femoral head. J Res Med Sci 2017; 22: 15.

4. Okazaki S, Nagoya S, Matsumoto H, Mizuo K, Sasaki M, Watanabe S, Yamashita $T$, Inoue $H$. Development of nontraumatic osteonecrosis of the femoral head requires toll-like receptor 7 and 9 stimulations and is boosted by repression on nuclear factor kappa $B$ in rats. Lab Invest 2015; 95(1): 92-99.

5. Moya-Angeler J, Gianakos AL, Villa JC, Ni A, Lane JM. Current concepts on osteonecrosis of the femoral head. World J Orthop 2015; 6(8): 590-601.

6. Wang C, Wang Y, Meng HY, Yuan XL, Xu XL, Wang AY, Guo QY, Peng J, Lu SB. Application of bone marrow mesenchymal stem cells to the treatment of osteonecrosis of the femoral head. Int $J$ Clin Exp Med 2015; 8(3): 3127-3135.

Trop J Pharm Res, March 2021; 20(3): 571 
7. O'Brien J, Hayder H, Zayed Y, Peng C. Overview of MicroRNA Biogenesis, Mechanisms of Actions, and Circulation. Front Endocrinol (Lausanne) 2018; 9: 402.

8. Filipowicz $W$, Bhattacharyya $S N$, Sonenberg $N$. Mechanisms of post-transcriptional regulation by microRNAs: are the answers in sight? Nat Rev Genet 2008; 9(2): 102-114.

9. Jia J, Feng $X, X u W$, Yang S, Zhang Q, Liu X, Feng $Y$, Dai Z. MiR-17-5p modulates osteoblastic differentiation and cell proliferation by targeting SMAD7 in nontraumatic osteonecrosis. Exp Mol Med 2014; 46(7): e107.

10. $X u$ W, Li J, Tian $H$, Wang $R$, Feng Y, Tang J, Jia J. MicroRNA-186-5p mediates osteoblastic differentiation and cell viability by targeting CXCL13 in non-traumatic osteonecrosis. Mol Med Rep 2019; 20(5): 4594-4602.

11. Dai Z, Jin Y, Zheng J, Liu K, Zhao J, Zhang S, Wu F, Sun Z. MiR-217 promotes cell proliferation and osteogenic differentiation of BMSCs by targeting DKK1 in steroidassociated osteonecrosis. Biomed Pharmacother 2019; 109: 1112-1119.

12. Wei $B$, Wei W. Identification of aberrantly expressed of serum microRNAs in patients with hormone-induced nontraumatic osteonecrosis of the femoral head. Biomed Pharmacother 2015; 75: 191-195.

13. Association WM. World Medical Association Declaration of Helsinki. Ethical principles for medical research involving human subjects. Bulletin of The World Health Organization 2001; 79(4): 373.

14. Zhao D, Zhang F, Wang B, Liu B, Li L, Kim SY, Goodman $S B$, Hernigou $P$, Cui $Q$, Lineaweaver $W C$ et al. Guidelines for clinical diagnosis and treatment of osteonecrosis of the femoral head in adults (2019 version). J Orthop Translat 2020; 21: 100-110.

15. Fu L, Liu H, Lei W. MiR-596 inhibits osteoblastic differentiation and cell proliferation by targeting Smad3 in steroid-induced osteonecrosis of femoral head. J Orthop Surg Res 2020; 15(1): 173.

16. Tang $X$, Zeng $X$, Huang $Y$, Chen $S$, Lin F, Yang G, Yang $N$. miR-423-5p serves as a diagnostic indicator and inhibits the proliferation and invasion of ovarian cancer. Exp Ther Med 2018; 15(6): 4723-4730.

17. Sun G, Ding $X, B i N$, Wu L, Wang J, Zhang W, Dong $X$, Lv $N$, Song $Y$, Zhan $Q$ et al. MiR-423-5p in brain metastasis: potential role in diagnostics and molecular biology. Cell Death Dis 2018; 9(10): 936.

18. Yang W, Wang J, Chen Z, Chen J, Meng $Y$, Chen L, Chang $Y$, Geng B, Sun L, Dou $L$ et al. NFE2 Induces miR-423-5p to Promote Gluconeogenesis and Hyperglycemia by Repressing the Hepatic FAM3A-ATPAkt Pathway. Diabetes 2017; 66(7): 1819-1832.

19. Hjelmeland $A B$, Schilling $S H$, Guo $X$, Quarles $D$, Wang $X F$. Loss of Smad3-mediated negative regulation of Runx2 activity leads to an alteration in cell fate determination. Mol Cell Biol 2005; 25(21): 9460-9468.

20. Huang $Y$, Zheng Y, Jia L, Li W. Long Noncoding RNA H19 Promotes Osteoblast Differentiation Via TGFB1/Smad3/HDAC Signaling Pathway by Deriving miR675. Stem Cells 2015; 33(12): 3481-3492.

21. Hao C, Yang S, Xu W, Shen JK, Ye S, Liu X, Dong Z, Xiao B, Feng Y. MiR-708 promotes steroid-induced osteonecrosis of femoral head, suppresses osteogenic differentiation by targeting SMAD3. Sci Rep 2016; 6: 22599. 